Journal of Applied Pharmaceutical Science Vol. 5 (11), pp. 121-126, November, 2015

Available online at http://www.japsonline.com

DOI: $10.7324 / J A P S .2015 .501121$

ISSN 2231-3354 (cc) BY-NC-SA

\title{
Synthesis and in vitro Anti Microbial Evaluation Including Anti- Malarial Activity of Pyrazole Based Novel Cinnoline Derivatives
}

\author{
S. Hurmath Unnissa*, Nilofer Nisha, Gayam Krishna Reddy \\ Department of Pharmaceutical Chemistry, KMCH College Of Pharmacy, Coimbatore, India.
}

\begin{tabular}{|c|c|}
\hline ARTICLE INFO & ABSTRACT \\
\hline $\begin{array}{l}\text { Article history: } \\
\text { Received on: } 26 / 08 / 2015 \\
\text { Revised on: } 14 / 09 / 2015 \\
\text { Accepted on: } 09 / 10 / 2015 \\
\text { Available online: } 27 / 11 / 2015\end{array}$ & $\begin{array}{l}\text { A Series of 4-methyl-3-[5-(substituted phenyl)-4, 5-dihydro-1H-Pyrazol-3-yl] Cinnoline-6-Sulfonamide were } \\
\text { synthesized from 4-methyl-3-acetylcinnoline-6-Sulfonamido chalcones and hydrazines. The structure of the } \\
\text { synthesized compounds were characterized by UV, IR, NMR \& Mass spectral data, and evaluated for their in } \\
\text { vitro anti-malarial and anti-bacterial activity to get new congeners as analogs of Pyrazole based Cinnoline } \\
\text { compounds as a potent anti-Malarial and anti-microbial agents. All analogues exhibited in vitro anti-malarial }\end{array}$ \\
\hline $\begin{array}{l}\text { Key words: } \\
\text { Cinnoline, pyrazole, } \\
\text { Plasmodium falciparum, } \\
\text { anti-bacterial, chalcones. }\end{array}$ & pathogenic microbes. \\
\hline
\end{tabular}

\section{INTRODUCTION}

Malaria is the most dreadful illness and widespread infectious disease because of its prevalence, virulence and drug resistance, having an overwhelming impact on public health in developing regions of the world. It affects more than 2400 million people, over $40 \%$ of the world population. Plasmodium falciparum is the main cause of severe clinical malaria and the World Health Organization (WHO) has forecast an annual growth of $16 \%$ in malarial cases. As the parasites rapidly develop permanent resistance against the different subclasses of existing drugs, there is a great urge to develop new and effective drugs attacking crucial targets in the metabolism of the malaria pathogen. Microorganisms like bacteria causes many infections like meningitis, ottis media, pneumonia, cholera, food poisoning, urinary tract infections. Many of these diseases are fatal if untreated, and treatment has been complicated by the resistance of the microorganisms to the widely used drugs. To combat the

\footnotetext{
* Corresponding Author

Mrs. S. Hurmath unnissa, Dept of Pharmaceutical chemistry,

KMCH College Of Pharmacy, Coimbatore-48, India.

Email:nissakmch@gmail.com
}

problem of resistance newer drugs are needed. Cinnoline is a versatile lead molecule that has been investigated widely in medicinal chemistry due to its important pharmacological activities (Gautam 2010, Eman et al., 2012, Coudert, 1991, Tonk, 2012).

It have been reported to exhibit anti-microbial, antitubercular, anti-cancer, anti-malarial, anti-hypertensive, antipyretic, anti-thrombolytic, analgesic, anti-diabetic, anti-depressant, cardiotonic, anaesthetic, anxiolytic etc. Cinnoline ring system is an isosteric relative to either Quinoline or Isoquinoline ( $\mathrm{Li}$ et al., 2013), therefore, in many cases the synthesized Cinnoline compounds were designed as analogs of Quinoline or isoquinoline. Cinoxacin is a cinnoline analogue of the Quinoline antibacterials used for urinary tract infection. Cinnoline is an isosteric analogue of quinoline and best can exhibit anti-malarial activity (Eman et al., 2012).

Pyrazoles and their derivatives have gained considerable importance over the years due to their wide range of biological activities like antibacterial, anticancer, anti-inflammatory, antitumor, anticonvulsant (Wentland, 2013). This prompted us in the synthesis of new congeners as analogs of pyrazole based Cinnoline compounds to get potent anti-microbial agents. 


\section{MATERIALS AND METHODS}

\section{Synthesis of Phenyl Hydrazano Acetyl Acetone-4-Sulfonamide} (Gautam, 2010)

Sodium nitrite $(7.4 \mathrm{gm}, 0.1 \mathrm{~mol})$ dissolved in $26 \mathrm{ml}$ of water was added to a suspension of Sulfanilamide $(10 \mathrm{gm}, 0.1 \mathrm{~mol})$ in $1 \mathrm{~N} \mathrm{HCl}(200 \mathrm{ml})$, and the mixture was stirred for $1 \mathrm{hr}$ at $0-5^{\circ} \mathrm{C}$ and filtered to obtain the clear diazonium salt.

The diazonium salt obtained was then added to a well stirred solution of ethanol $(30 \mathrm{ml})$, water $(500 \mathrm{ml})$ and acetyl acetone $(10.01 \mathrm{gm}, 0.1 \mathrm{~mol})$ at $0^{\circ} \mathrm{C}$ with stirring. Sodim acetate was then added to keep the mixture alkaline to litmus after 3 hour stirring at $0^{\circ} \mathrm{C}$ the crude product was filtered, washed with water and air dried. Recrystallisation from ethanol afforded yellow needles of purified Phenyl hydrazano acetyl acetone-4Sulfonamide.

\section{Synthesis of 4-methyl 3-acetyl Cinnoline 6-Sulfonamide}

The Phenyl hydrazano acetyl acetone-4-Sulfonamide $(10 \mathrm{~g}, 0.05 \mathrm{~mole})$ was added to the Polyphosphoric acid $(16 \mathrm{gm}$, $7.216 \mathrm{ml}, 0.03$ mole) in small lots over 30 mins while maintaining the temperature between $60-65^{\circ} \mathrm{C}$. The reaction was maintained for an additional 2 hour and monitored by TLC. After the completion of reaction, ice cold water $(200 \mathrm{ml})$ was added carefully to decompose the black residue at $0-5^{\circ} \mathrm{C}$. The product was then extracted with ethyl acetate. Ethyl acetate layer was then treated with Charcoal and concentrated to get the crude product as a brownish black residue. Recrystallisation from methanol to obtained as light yellow crystals of 4-methyl 3-acetyl Cinnoline 6Sulfonamide.

Synthesis of 1-(4-methyl Cinnoline-3-yl)-3-(substituted phenyl) prop-2-en-1-one 6-Sulfonamide [Cinnoline based chalcone]

The product obtained from step $3(2.03 \mathrm{gm}, 0.01 \mathrm{~mole})$ and aromatic aldehyde in same ratio $(0.01 \mathrm{~mole})$ in ethanol $(50 \mathrm{ml})$ was cooled at $0-5^{\circ} \mathrm{C}$ and added $(5-10 \mathrm{ml}) 40 \% \mathrm{NaOH}$ solution till precipitated and washed with ice water. Few drops N/20 dilute $\mathrm{HCl}$ was added for complete precipitation and filtered, washed with ice water and recrystalized from alcohol to afford the compound (CN-1 -11).

\section{Synthesis of 3-(4'-methyl-(3"-Cinnolinyl)-5-(substituted phenyl)-1H-Pyrazoline 6-Sulfonamide (CN 1a-11a)}

The compound $\mathrm{CN}-1-11(0.01 \mathrm{~mole})$ in $20 \mathrm{ml}$ acetic acid was taken and hydrazine hydrate $(0.01 \mathrm{~mole})$ was added to it and refluxed for 10 hour. The contents were poured into ice, filtered and the product isolated, crystallized from ethanol to afford the compound (CN-1a-11a). The purity of the products were confirmed by a single spot on the TLC plate and solvent system used was Benzene:Ethyl acetate (8:2). Melting point was determined and uncorrected. The physicochemical properties of synthesized compounds are given in table: 1

Table: 1 Physicochemical properties of synthesised compounds.

\begin{tabular}{|c|c|c|c|c|c|c|c|c|c|}
\hline S.N & $\begin{array}{c}\text { Compound } \\
\text { code }\end{array}$ & $\mathbf{R}$ & $\begin{array}{c}\text { Molecular } \\
\text { formula }\end{array}$ & \begin{tabular}{c}
\multicolumn{2}{c}{$\begin{array}{c}\text { Molecular } \\
\text { weight } \quad(g)\end{array}$} \\
\end{tabular} & $\begin{array}{c}\text { Percentage } \\
\text { yield }(\%)\end{array}$ & Colour & Solubility & $\begin{array}{c}\text { Melting } \\
\text { Point }\left({ }^{0} \mathrm{C}\right) \\
\end{array}$ & $\mathbf{R}_{\mathrm{f}}$ value \\
\hline 1 & $\mathrm{CN}-1 \mathrm{a}$ & & $\mathrm{C}_{18} \mathrm{H}_{16} \mathrm{~N}_{6} \mathrm{O}_{4} \mathrm{~S}$ & 412.42 & $92.5 \%$ & Yellow & DMSO & $82^{\circ} \mathrm{C}$ & 0.68 \\
\hline 2 & $\mathrm{CN}-2 \mathrm{a}$ & & $\mathrm{C}_{18} \mathrm{H}_{17} \mathrm{~N}_{5} \mathrm{O}_{3} \mathrm{~S}$ & 388.42 & $86.1 \%$ & Yellow & DMSO & $110^{\circ} \mathrm{C}$ & 0.72 \\
\hline 3 & $\mathrm{CN}-3 \mathrm{a}$ & & $\mathrm{C}_{18} \mathrm{H}_{16} \mathrm{~N}_{5} \mathrm{O}_{2} \mathrm{~S}$ & 366.42 & $87.2 \%$ & Yellow & DMSO & $85^{\circ} \mathrm{C}$ & 0.71 \\
\hline 4 & $\mathrm{CN}-4 \mathrm{a}$ & & $\mathrm{C}_{19} \mathrm{H}_{19} \mathrm{~N}_{5} \mathrm{O}_{3} \mathrm{~S}$ & 397.45 & $90.7 \%$ & Yellow & DMSO & $87^{\circ} \mathrm{C}$ & 0.63 \\
\hline 5 & $\mathrm{CN}-5 \mathrm{a}$ & & $\mathrm{C}_{19} \mathrm{H}_{19} \mathrm{~N}_{5} \mathrm{O}_{4} \mathrm{~S}$ & 413.45 & $92.2 \%$ & Yellow & DMSO & $82^{\circ} \mathrm{C}$ & 0.52 \\
\hline 6 & $\mathrm{CN}-6 \mathrm{a}$ & & $\mathrm{C}_{20} \mathrm{H}_{19} \mathrm{~N}_{5} \mathrm{O}_{2} \mathrm{~S}$ & 393.46 & $86.1 \%$ & $\begin{array}{l}\text { Golden } \\
\text { brown }\end{array}$ & DMSO & $80^{\circ} \mathrm{C}$ & 0.67 \\
\hline 7 & $\mathrm{CN}-7 \mathrm{a}$ & & $\mathrm{C}_{20} \mathrm{H}_{22} \mathrm{~N}_{6} \mathrm{O}_{2} \mathrm{~S}$ & 410.49 & $91.4 \%$ & Yellow & DMSO & $210^{\circ} \mathrm{C}$ & 0.75 \\
\hline 8 & $\mathrm{CN}-8 \mathrm{a}$ & & $\mathrm{C}_{18} \mathrm{H}_{16} \mathrm{FN}_{5} \mathrm{O}_{2} \mathrm{~S}$ & 385.42 & $76.2 \%$ & Yellow & DMSO & $92^{\circ} \mathrm{C}$ & 0.62 \\
\hline 9 & CN-9a & & $\mathrm{C}_{18} \mathrm{H}_{16} \mathrm{ClN}_{5} \mathrm{O}_{2} \mathrm{~S}$ & 401.87 & $82.4 \%$ & Yellow & DMSO & $85^{\circ} \mathrm{C}$ & 0.66 \\
\hline 10 & $\mathrm{CN}-10 \mathrm{a}$ & & $\mathrm{C}_{18} \mathrm{H}_{16} \mathrm{ClN}_{5} \mathrm{O}_{2} \mathrm{~S}$ & 401.87 & $74.2 \%$ & $\begin{array}{l}\text { Brownish } \\
\text { yellow }\end{array}$ & DMSO & $87^{\circ} \mathrm{C}$ & 0.56 \\
\hline 121 & $\mathrm{CN}-11 \mathrm{a}$ & & $\mathrm{C}_{18} \mathrm{H}_{16} \mathrm{~N}_{6} \mathrm{O}_{4} \mathrm{~S}$ & 412.42 & $89.5 \%$ & Yellow & DMSO & $112^{\circ} \mathrm{C}$ & 0.58 \\
\hline
\end{tabular}



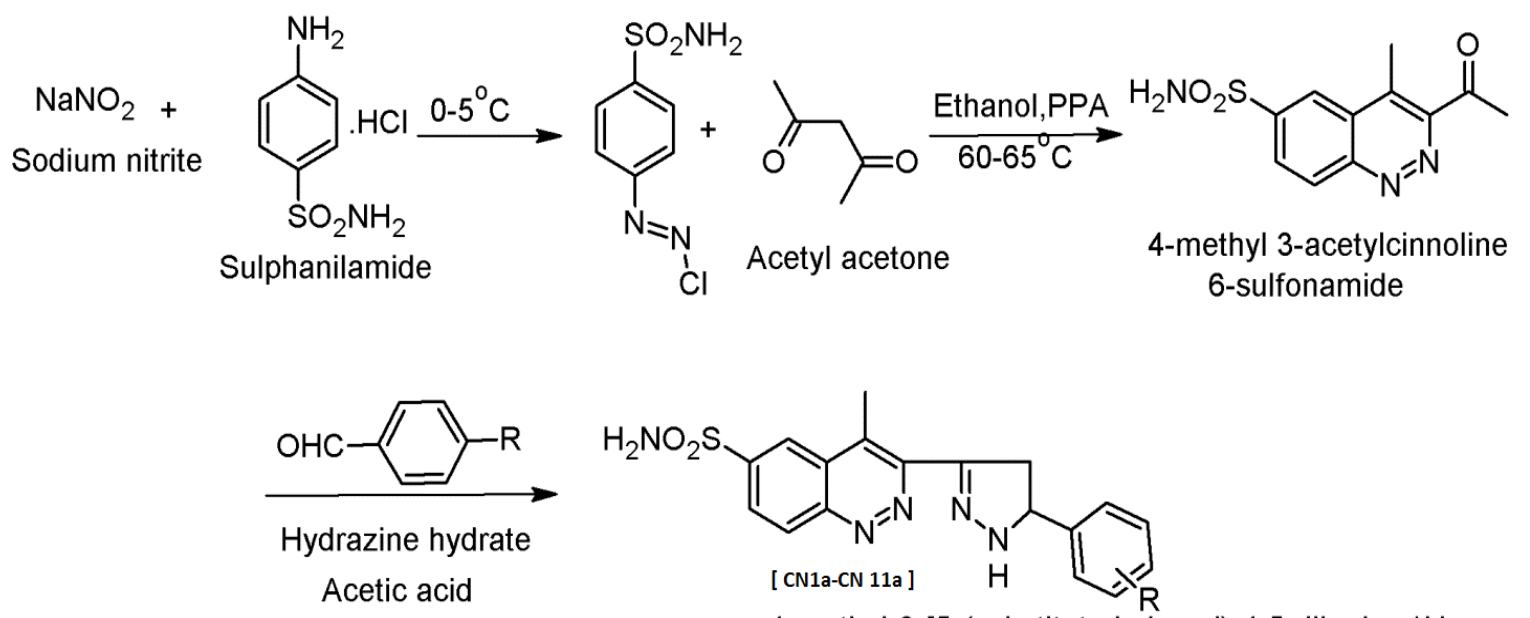

SCHEME

4-methyl-3-[5-(substituted phenyl)-4.5-dihydro-1H-

Pyrazol-3-yl]cinnoline-6-sulfonamide

4-methyl-3-[5-(4-nitrophenyl)-4,5-dihydro-1H- Pyrazol-3-

yl]Cinnoline-6-sulfonamide

$\mathrm{IR}(\mathrm{KBr}, \mathrm{cm} 1), 3126.04(\mathrm{NH}), 2925\left(\mathrm{CH}_{3}\right), 1503.24(\mathrm{C}=\mathrm{N})$, $1356.08\left(\mathrm{NO}_{2}\right), 1302.08(\mathrm{~S}=0), \quad 1164.79(\mathrm{C}-\mathrm{N}), \quad 846.59(\mathrm{p}$-subs benzene), 681.713(C-S), 1HNMR( $\delta \mathrm{ppm}) ; 8.37-8.63(\mathrm{~m}, \mathrm{ArH})$, $2.35\left(\mathrm{~s}, 2 \mathrm{H}\right.$ in $\left.\mathrm{CH}_{2}\right), 2.0\left(\mathrm{~s}, 2 \mathrm{H}\right.$ in $\left.\mathrm{NH}_{2}\right), 2.55\left(\mathrm{~s}, 3 \mathrm{H}, \mathrm{CH}_{3}\right), \mathrm{MS}(\mathrm{m} / \mathrm{z})$ : 198.99 .

\section{4-methyl-3-[5-(4-hydroxyphenyl )-4,5-dihydro-1H-Pyrazol-3- yl]Cinnoline-6-sulfonamide}

3126.04(N-H), 2925(CH3), 1503.24(C=N), 3000(Br OH group) $1302.08(\mathrm{~S}=0), 1164.79(\mathrm{C}-\mathrm{N}), 800-900(\mathrm{p}$-subs benzene), 681.713(C-S), 1HNMR( $\delta \mathrm{ppm}): 8.21-8.34(\mathrm{~m}, \quad$ ArH $) \quad 7.2(\mathrm{~s}, \mathrm{NH})$ $1.9(\mathrm{~s}, 2 \mathrm{H}$ inCH2) $2.35(\mathrm{~s}, 3 \mathrm{H}$ inCH3) $2(\mathrm{~s}, 2 \mathrm{H}$ in NH2) $6.68-6.95(\mathrm{~m}$, ArH). MS (m/z)m+:389

\section{4-methyl-3-[5-(4-chlorophenyl) -4,5-dihydro-1H-Pyrazol-3-}

yl]Cinnoline-6-sulfonamide

IR(KBr,cm1): $3126.04(\mathrm{~N}-\mathrm{H}), 2925\left(\mathrm{CH}_{3}\right), 1503.24(\mathrm{C}=\mathrm{N})$, 1400.07(C-Cl), 1164.79(C-N) 810.17(p-subs benzene), 681.713(CS). 1HNMR $(\delta p p m) ; 8.1-8.03(\mathrm{~m}, \mathrm{ArH}), 2.35\left(\mathrm{~s}, 2 \mathrm{H}\right.$ in $\left.\mathrm{CH}_{2}\right)$, 2.0(s,2H in $\left.\mathrm{NH}_{2}\right), 2.55\left(\mathrm{~s}, 3 \mathrm{H}, \mathrm{CH}_{3}\right), \mathrm{MS}(\mathrm{m} / \mathrm{z}) \mathrm{m}+: 367.99$.

\section{4-methyl-3-[5-(4-methoxyphenyl)-4,5-dihydro-1H-Pyrazol-3-}

yl]Cinnoline-6-sulfonamide

IR(KBr,cm1): 3126.04(N-H), 2925( $\left.\mathrm{CH}_{3}\right), 1503.24(\mathrm{C}=\mathrm{N})$, $1312.57\left(\mathrm{OCH}_{3}\right), \quad 1164.79(\mathrm{C}-\mathrm{N}) \quad 810.17$ (p-subs benzene), 681.713(C-S), 1HNMR( $\delta \mathrm{ppm}): 8.21-8.55(\mathrm{~m}, 4 \mathrm{H}, \mathrm{CH}$ in Cinnoline) $7(\mathrm{~s}, \mathrm{NH}) \quad 1.9(\mathrm{~s}, 2 \mathrm{H}, \mathrm{CH} 2 \quad) \quad 2.35(\mathrm{~s}, 3 \mathrm{H}, \mathrm{CH} 3 \quad) \quad 2.0(\mathrm{~s}, 2 \mathrm{H}, \mathrm{NH} 2)$ $3.7\left(\mathrm{~s}, 3 \mathrm{H}, \mathrm{OCH}_{3}\right)$. MS (m/z) 398

\footnotetext{
4-methyl-3-[5-(4-hydroxy-3-methoxyphenyl)- 4,5-dihydro-1HPyrazol-3-yl]Cinnoline-6-sulfonamide

IR(KBr,cm1): $\quad 3126.04(\mathrm{~N}-\mathrm{H}), 3111.58$ (OHGroup), 2925(CH3), $1503.24(\mathrm{C}=\mathrm{N}) \quad 1334.45(\mathrm{OCH} 3 \mathrm{Bend}), 1188.9$
}

(C-C),1164.79(C-N),810.17 (p-subsbenzene), 681.713(C-S), 1HNMR (8ppm): $8.21-8.55(\mathrm{~m}, \mathrm{CH}$ in Cinnoline) $7(\mathrm{~s}, \mathrm{NH})$ $1.9\left(\mathrm{~s}, 2 \mathrm{H}, \mathrm{CH}_{2}\right) 2.35\left(\mathrm{~s}, 3 \mathrm{H}, \mathrm{OCH}_{3}\right) \quad 2\left(\mathrm{~s}, 2 \mathrm{H}, \mathrm{NH}_{2}\right) \quad 2.2\left(\mathrm{~s}, 3 \mathrm{H}, \mathrm{CH}_{3}\right) 5$ $(\mathrm{s}, 1 \mathrm{H}, \mathrm{OH}$ in aromatic), $6.56-6.75(\mathrm{~m}, 4 \mathrm{H}, \mathrm{ArH}) . \mathrm{MS}(\mathrm{m} / \mathrm{z}) 414$

\section{4-methyl-3-\{5-[(E)-2-phenylethenyl]-4,5- dihydro-1H-Pyrazol-3- yljCinnoline-6-sulfonamide $\mathrm{IR}(\mathrm{KBr}, \mathrm{cm} 1): \quad 3126.04(\mathrm{~N}-\mathrm{H}) \quad 2925\left(\mathrm{CH}_{3}\right) \quad 1631(\mathrm{C}=\mathrm{C})$ 1503.24(C=N) 1188.9(C-C) 1164.79(C-N) 710.14(Mono subs benzene) $\quad 681.713(\mathrm{C}-\mathrm{S}) .1 \mathrm{HNMR}(\mathrm{\delta ppm}): \quad 8.1-8.4(\mathrm{~m}, \quad \mathrm{ArH})$ 7.2(s, NH) $1.9(\mathrm{~s}, 2 \mathrm{H}$ inCH2) $2.35(\mathrm{~s}, 3 \mathrm{H}$ inCH3) $2(\mathrm{~s}, 2 \mathrm{H}$ in $\mathrm{NH} 2)$ 6.68-6.95(m, ArH). MS (m/z)m+:393}

\section{4-methyl-3-[5-(4-dimethylphenyl)-4,5- dihydro-1H-Pyrazol-3- yl]Cinnoline-6-sulfonamide}

IR(KBr,cm1): $3200($ Secondary amine), $3126.04(\mathrm{~N}-\mathrm{H})$, $2925\left(\mathrm{CH}_{3}\right), 1503.24(\mathrm{C}=\mathrm{N}), 1188.9(\mathrm{C}-\mathrm{C}) \quad 1164.79(\mathrm{C}-\mathrm{N}), 846.597(\mathrm{p}-$ subsbenzene),681.713(C-S),1HNMR( $(\mathrm{ppm}) 8.21 .55(\mathrm{~m}, \mathrm{ArH}) 7(\mathrm{NH})$ $1.9(\mathrm{~s}, 2 \mathrm{H}, \mathrm{CH} 2) \quad 2.35(\mathrm{~s}, 3 \mathrm{H}, \mathrm{CH} 3) \quad 2(\mathrm{~s}, 2 \mathrm{H}, \mathrm{NH} 2) \quad 6.54-$ 6.94(m,4H,ArH). MS (m/z)411

\section{4-methyl-3-[5-(3-fluorophenyl)-4,5 -dihydro-1H-Pyrazol-3- yl]Cinnoline-6-sulfonamide}

IR( $\mathrm{KBr}, \mathrm{cm} 1): \quad 3200$ (Secondary amine) $3126.04(\mathrm{~N}-\mathrm{H})$ $2925\left(\mathrm{CH}_{3}\right)$ 1503.24(C=N) 1400(C-F) 1188.9(C-C) 1164.79(C-N) 730-750(m-subs benzene) 681.713(C-S). 1HNMR( $\delta \mathrm{ppm})$ : 8.298.3(m, $\quad \operatorname{ArH}) \quad 7.2(\mathrm{~s}, \mathrm{NH}) \quad 1.89(\mathrm{~s}, \quad 2 \mathrm{H} \quad \mathrm{inCH} 2) \quad 2.31$ $(\mathrm{s}, 3 \mathrm{H}$ inCH3 $) \quad 2(\mathrm{~s}, 2 \mathrm{H}$ in $\mathrm{NH} 2) \quad 6.68-6.95(\mathrm{~m}, \mathrm{ArH}) . \quad \mathrm{MS}$ $(\mathrm{m} / \mathrm{z}) \mathrm{m}+: 386$.

\section{4-methyl-3-[5-(2-chlorophenyl)-4,5- dihydro-1H-Pyrazol-3- yl]Cinnoline-6-sulfonamide}

$\mathrm{IR}(\mathrm{KBr}, \mathrm{cm} 1): 3126.04(\mathrm{~N}-\mathrm{H}) \quad 2925\left(\mathrm{CH}_{3}\right) \quad 1503.24(\mathrm{C}=\mathrm{N})$ $1401.03(\mathrm{C}-\mathrm{Cl}) \quad 1188.9(\mathrm{C}-\mathrm{C}) \quad 1164.79(\mathrm{C}-\mathrm{N}) \quad 681.713(\mathrm{C}-\mathrm{S})$ 670.142(O-subs benzene). 1HNMR( $\delta \mathrm{ppm}): 8.01-8.14(\mathrm{~m}, \mathrm{ArH})$ 
7.2(s, NH) $1.91(\mathrm{~s}, 2 \mathrm{H}$ inCH2) $2.36(\mathrm{~s}, 3 \mathrm{H}$ inCH3) $2(\mathrm{~s}, 2 \mathrm{H}$ in $\mathrm{NH} 2)$ 6.68-6.95(m, ArH). MS (m/z)m+:402. 4-methyl-3-[5-(3-chlorophenyl)-4,5- dihydro-1H-Pyrazol-3-
yl]Cinnoline-6-sulfonamide

$\mathrm{IR}(\mathrm{KBr}, \mathrm{cm} 1): 3126.04(\mathrm{~N}-\mathrm{H}) 2925(\mathrm{CH} 3) \quad 1503.24(\mathrm{C}=\mathrm{N})$ 1401.03(C-Cl) $\quad 1188.9(\mathrm{C}-\mathrm{C}) \quad 1164.79(\mathrm{C}-\mathrm{N}) \quad 730-780(\mathrm{~m}-\mathrm{subs}$

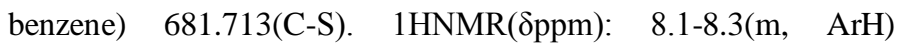
7.(s,NH) $1.99(\mathrm{~s}, 2 \mathrm{H}$ inCH2) $2.35(\mathrm{~s}, 3 \mathrm{H}$ inCH3) $2(\mathrm{~s}, 2 \mathrm{H}$ in $\mathrm{NH} 2)$ 6.68-6.95(m, ArH). MS (m/z)m+:402

\section{4-methyl-3-[5-(2-nitrophenyl)-4,5-dihydro -1H-Pyrazol-3- yl]Cinnoline-6-sulfonamide}

3126.04(N-H) 2925(CH3) 1530.24( $\left.\mathrm{NO}_{2}\right) 1503.24(\mathrm{C}=\mathrm{N})$ 1188.9(C-C) 1164.79(C-N) 1188.9(C-C) 680-749(o-subs benzene) 681.713(C-S) $\quad$ 1HNMR( $\delta \mathrm{ppm}): \quad 8.21-8.34(\mathrm{~m}, \quad$ ArH $) \quad 7 .(\mathrm{s}, \mathrm{NH})$ $1.87(\mathrm{~s}, 2 \mathrm{H}$ inCH2) $2.3(\mathrm{~s}, 3 \mathrm{H}$ inCH3) $2(\mathrm{~s}, 2 \mathrm{H}$ in $\mathrm{NH} 2) 6.8-6.9(\mathrm{~m}$, $\mathrm{ArH})$. MS $(\mathrm{m} / \mathrm{z}) \mathrm{m}+. . \mathrm{m} / \mathrm{z}(\mathrm{m}+)=413$

\section{ANTI MALARIAL SCREENING: CANDLE JAR METHOD}

Plasmodium falciparum (ATCC 30932, FCR-3 strain) was cultivated by the method of (Trager, 1976) using a 5\% hematocrit of type Human red blood cells suspended in a RPMI 1640 mediums, and supplemented with heat-activated $10 \%$ type $\mathrm{A}$ human serum. The plates were placed in a $\mathrm{CO}_{2}-\mathrm{O}_{2}-\mathrm{N}_{2}$ incubator $\left(5 \% \mathrm{CO}_{2}, 5 \% \mathrm{O}_{2}\right.$ and $90 \% \mathrm{~N}_{2}$ atmosphere) at $37{ }^{\circ} \mathrm{C}$, and the medium was changed daily until $5 \%$ parasitemia (which means the existence of 5 parasite-infected erythrocytes in every 100 erythrocytes) (Collins, 1997).

Table 2: In vitro anti plasmodial activity of synthesized compounds against plasmodium falciparum

\begin{tabular}{cccccc}
\hline S.No & $\begin{array}{c}\text { Compound } \\
\text { Code }\end{array}$ & \multicolumn{4}{c}{ IC $_{\mathbf{5 0}}$ Values $(\%)$} \\
\cline { 3 - 6 } & & $\begin{array}{c}\mathbf{2 0} \\
(\boldsymbol{\mu g} / \mathbf{m l})\end{array}$ & $\begin{array}{c}\mathbf{4 0} \\
(\boldsymbol{\mu} \mathbf{g} / \mathbf{m l})\end{array}$ & $\begin{array}{c}\mathbf{6 0} \\
(\boldsymbol{\mu g} / \mathbf{m l})\end{array}$ & $\begin{array}{c}\mathbf{8 0} \\
(\boldsymbol{\mu g} / \mathbf{m l})\end{array}$ \\
\hline $\mathbf{1}$ & Pyremethamine & 72.30 & 80.15 & 87.69 & 95.32 \\
$\mathbf{2}$ & CN-1a & 59.23 & 66.92 & 74.61 & 82.30 \\
$\mathbf{3}$ & CN-2a & 46.15 & 51.53 & 66.92 & 72.30 \\
$\mathbf{4}$ & CN-3a & 36.15 & 46.15 & 53.84 & 61.53 \\
$\mathbf{5}$ & CN-4a & 49.23 & 56.92 & 64.61 & 72.30 \\
$\mathbf{6}$ & CN-5a & 69.23 & 76.92 & 84.61 & 87.69 \\
$\mathbf{7}$ & CN-6a & 30.76 & 38.46 & 43.84 & 50.00 \\
$\mathbf{8}$ & CN-7a & 41.53 & 46.15 & 57.69 & 61.53 \\
$\mathbf{9}$ & CN-8a & 52.30 & 61.53 & 69.23 & 76.92 \\
$\mathbf{1 0}$ & CN-9a & 27.69 & 42.30 & 51.53 & 56.92 \\
$\mathbf{1 1}$ & CN-10a & 33.84 & 43.84 & 61.53 & 72.30 \\
$\mathbf{1 2}$ & CN-11a & 61.53 & 69.23 & 76.92 & 84.61 \\
\hline
\end{tabular}

Various concentrations of the synthesized compounds and standard drug pyremethamine $(20,40,60,80 \mu \mathrm{g} / \mathrm{ml})$ in DMSO, was added to the well plates containing $0.5 \mathrm{ml}$ of the culture mediums, $20 \mu \mathrm{l}$ of washed erythrocytes and $20 \mu \mathrm{l}$ of P.falciparum infected human blood. Well plates were placed in the candle jar and incubated at $37^{\circ} \mathrm{C}$ for $24 \mathrm{hrs}$. Parasite counts were made on the giemsa stained thin smears prepared at different time intervals $(24,48,72 \mathrm{hrs})$. And in vitro anti-plasmodial activity of the synthesized compounds was determined by calculating inhibitory concentration percentage. The values are given in table 2.

Percentage can be calculated using the formula,

$\mathbf{I C}_{\mathbf{5 0}=}=$ No of parasitemia in control - No of parasitemia in treated $\times 100$ No of parasitemia in control

\section{Anti bacterial screening: Disc diffusion method}

Muller Hinton agar medium were prepared and transferred into the sterile Petri plates aseptically (thickness of 5$6 \mathrm{~mm}$ ). Standardized bacterial inoculums of Micrococcus luteus, Staphylococcus aureus, Bacillus subtilis, Corynebacterium diphtheria, Bacillus linctus, Escherichia coli, Pseudomonas aureginosa, Rhodosporum rubrum, Vibrio cholera, Salmonella paratyphi were applied to the plates. The sample impregnated discs $(100 \mu \mathrm{g} / \mathrm{disc})$ in dimethyl sulphoxide and standard ciprofloxacin $10 \mu \mathrm{g}$ disc were placed on the inoculated agar medium. All petri plates were incubated at $37^{\circ} \mathrm{C}$ for $24 \mathrm{hrs}$. After the incubation produced by the sample were measured. The antibacterial activity were evaluated by measuring zone if inhibition in $\mathrm{mm}$ and the MIC were determined by Serial dilution method. The zone of inhibition and MIC values are given in table: $3 \& 4$

\section{RESULT AND DISCUSSION}

Provoked by the biological activity of the Cinnoline and in view of ongoing search for the most potent anti-malarial agent, some novel 3,7 Di substituted derivatives of Cinnoline have been synthesized by Griess diazo reaction followed by intra molecular cyclisation to get chalcone based products which on Intermolecular cyclization afforded pyrazole based cinnoline derivatives and their anti-malarial and anti-microbial activity was studied. The anti-malarial studies was carried out with all synthesized Cinnoline derivatives in the concentration of $20 \mu \mathrm{g} / \mathrm{ml}$, $40 \mu \mathrm{g} / \mathrm{ml}, 60 \mu \mathrm{g} / \mathrm{ml}, 80 \mu \mathrm{g} / \mathrm{ml}$ in DMSO against Plasmodium falciparum by using Candle jar method. Pyremethamine of same concentrations were used as a standard. The anti-malarial activities of the compounds were evaluated by estimation of percentage of inhibition of parasitemia at different concentration. It could be seen that these newly synthesized derivatives of Cinnoline exhibit moderate to good anti-malarial activity. Out of the compounds synthesized, CN-5a was most potent which exhibit $87 \%$ inhibition at $80 \mu \mathrm{g} / \mathrm{ml}$ concentration. Other derivatives, which also showed inhibition more than $50 \%$ respectively. The antibacterial studies were carried out with all the synthesized Cinnoline derivatives against gram positive and gram negative bacteria. It could be seen that these newly synthesized derivatives of Cinnoline exhibit moderate to good anti-bacterial activity. Out of the compounds synthesized, CN-11a, CN-9a, CN-5a was most potent with zone of inhibition against Rhodosporum rubrum, Vibrio cholera and Escherichia coli. The MIC of the synthesized compounds against Micrococcus luteus, Staphylococcus aureus, Bacillus subtilis, Corynebacterium diphtheria, Bacillus linctus, Escherchia coli, Pseudomonas aureginosa, Rhodosporum rubrum, Vibrio cholera and Salmonella paratyphi was determined by serial dilution method, was found to be in the range of $1.2-2.5 \mu \mathrm{g} / \mathrm{ml}$. 
Table 3: Anti-bacterial activity [zone of inhibition] of the synthesized compounds by Disc Diffusion method.

\begin{tabular}{|c|c|c|c|c|c|c|c|c|c|c|c|c|c|}
\hline \multirow{3}{*}{$\begin{array}{l}\text { S. } \\
\text { No }\end{array}$} & \multirow{3}{*}{$\begin{array}{c}\text { Micro } \\
\text { organisms }\end{array}$} & \multicolumn{12}{|c|}{ Zone of Inhibition (in mm) } \\
\hline & & \multicolumn{12}{|c|}{ Compounds $(100 \mu \mathrm{g} / \mathrm{disc})$} \\
\hline & & $\begin{array}{l}\text { CN } \\
\text { 1a }\end{array}$ & $\begin{array}{l}\text { CN } \\
2 a\end{array}$ & $\begin{array}{l}\text { CN } \\
\text { 3a }\end{array}$ & $\begin{array}{c}\text { CN } \\
4 a\end{array}$ & $\begin{array}{c}\text { CN } \\
5 a\end{array}$ & $\begin{array}{c}\text { CN } \\
\text { 6a }\end{array}$ & $\begin{array}{l}\mathrm{CN} \\
7 \mathrm{a}\end{array}$ & $\begin{array}{l}\text { CN } \\
8 \mathrm{a}\end{array}$ & $\begin{array}{l}\text { CN } \\
9 a\end{array}$ & $\begin{array}{l}\text { CN } \\
10 a\end{array}$ & $\begin{array}{l}\text { CN } \\
11 a\end{array}$ & $\begin{array}{c}\text { Std } \\
*\end{array}$ \\
\hline 1 & M.luteus & 10 & 8 & 9 & 9 & 10 & 9 & 9 & 10 & 11 & 11 & 10 & 18 \\
\hline 2 & S.aureus & 11 & 10 & 12 & 8 & 11 & 12 & 12 & 11 & 10 & 11 & 11 & 17 \\
\hline 3 & B.subtilis & 9 & 9 & 10 & 9 & 9 & 10 & 10 & 9 & 9 & 9 & 10 & 15 \\
\hline 4 & C.diphtheria & 9 & 10 & 10 & 8 & 9 & 8 & 8 & 10 & 10 & 10 & 9 & 16 \\
\hline 5 & B.linctus & 8 & 10 & 8 & 10 & 10 & 11 & 11 & 11 & 9 & 9 & 11 & 18 \\
\hline 6 & E.coli & 11 & 10 & 12 & 11 & 10 & 11 & 11 & 11 & 13 & 15 & 12 & 16 \\
\hline 7 & P.aureginosa & 9 & 10 & 10 & 8 & 11 & 10 & 9 & 11 & 10 & 8 & 11 & 15 \\
\hline 8 & R.rubrum & 11 & 12 & 12 & 11 & 11 & 12 & 12 & 14 & 13 & 14 & 12 & 15 \\
\hline 9 & V.cholera & 9 & 11 & 11 & 10 & 14 & 11 & 13 & 11 & 14 & 10 & 15 & 18 \\
\hline 10 & S.paratyphi & 11 & 11 & 13 & 11 & 10 & 9 & 13 & 11 & 10 & 9 & 11 & 15 \\
\hline
\end{tabular}

*- Ciprofloxacin.

Table 4: Anti-bacterial activity[MIC] of the synthesized compounds by Serial Dilution method.

\begin{tabular}{|c|c|c|c|c|c|c|c|c|c|c|c|c|c|}
\hline \multirow[b]{2}{*}{$\begin{array}{l}\text { S } \\
\text { No. }\end{array}$} & \multirow{2}{*}{$\begin{array}{c}\text { Micro } \\
\text { organisms }\end{array}$} & \multicolumn{12}{|c|}{ MIC VALUES $(\mu \mathrm{g} / \mathrm{ml})$} \\
\hline & & $\begin{array}{c}\mathrm{CN} \\
\mathbf{1 a}\end{array}$ & $\begin{array}{c}\mathrm{CN} \\
2 \mathrm{a}\end{array}$ & $\begin{array}{l}\mathbf{C N} \\
\mathbf{3 a}\end{array}$ & $\begin{array}{l}\mathrm{CN} \\
\mathbf{4 a}\end{array}$ & $\begin{array}{c}\mathbf{C N} \\
5 \mathbf{a}\end{array}$ & $\begin{array}{c}\mathrm{CN} \\
6 \mathrm{a}\end{array}$ & $\begin{array}{c}\mathrm{CN} \\
7 \mathbf{a}\end{array}$ & $\begin{array}{l}\mathrm{CN} \\
\mathbf{8 a}\end{array}$ & $\begin{array}{c}\mathrm{CN} \\
9 \mathrm{a}\end{array}$ & $\begin{array}{l}\mathrm{CN} \\
10 \mathrm{a}\end{array}$ & $\begin{array}{l}\mathrm{CN} \\
11 \mathrm{a}\end{array}$ & Std* \\
\hline 1 & M.luteus & 1.2 & 5 & 2.5 & 2.5 & 1.2 & 2.5 & 2.5 & 1.2 & 1.2 & 2.5 & 1.2 & 1.2 \\
\hline 2 & S.aureus & 1.2 & 2.5 & 1.2 & 5 & 2.5 & 1.2 & 1.2 & 2.5 & 2.5 & 2.5 & 1.2 & 1.2 \\
\hline 3 & B.subtilis & 5 & 5 & 2.5 & 2.5 & 2.5 & 2.5 & 1.2 & 1.2 & 2.5 & 2.5 & 1.2 & 1.2 \\
\hline 4 & C.diphtheriae & 2.5 & 1.2 & 1.2 & 5 & 2.5 & 5 & 5 & 1.2 & 1.2 & 1.2 & 2.5 & 1.2 \\
\hline 5 & B.linctus & 5 & 2.5 & 5 & 2.5 & 2.5 & 2.5 & 1.2 & 1.2 & 2.5 & 2.5 & 1.2 & 1.2 \\
\hline 6 & E.coli & 1.2 & 2.5 & 1.2 & 2.5 & 1.2 & 5 & 1.2 & 1.2 & 1.2 & 2.5 & 2.5 & 1.2 \\
\hline 7 & P.aureginosa & 2.5 & 2.5 & 1.2 & 2.5 & 1.2 & 2.5 & 2.5 & 2.5 & 1.2 & 1.2 & 2.5 & 1.2 \\
\hline 8 & R.rubrum & 5 & 2.5 & 2.5 & 5 & 2.5 & 1.2 & 2.5 & 1.2 & 2.5 & 2.5 & 1.2 & 1.2 \\
\hline 9 & V.cholerae & 5 & 2.5 & 2.5 & 5 & 1.2 & 2.5 & 1.2 & 2.5 & 1.2 & 2.5 & 1.2 & 1.2 \\
\hline 10 & S.paratyphi & 2.5 & 2.5 & 1.2 & 2.5 & 2.5 & 5 & 1.2 & 2.5 & 2.5 & 2.5 & 2.5 & 1.2 \\
\hline
\end{tabular}

*-Ciprofloxacin.

\section{CONCLUSION}

In Summary, some novel substituted Cinnoline derivatives have been synthesized and evaluated for its antimalarial and anti-microbial activity. All derivatives demonstrated significant anti-malarial and anti-microbial activity amongst, compound CN-5a was found to be most potent compound with promising activity against resistant strains of Plasmodium falciparum, bacteria and fungus. Taking into account the significant activities of the examined compounds, it is believed that further optimization of these identified chemical leads can probably lead to the development of more active molecules. Further studies on its possible mechanism and invivo trials in experimental animals to broaden their pharmacological assessment, may provide a new analogue that can overcome drug resistance, prolonged treatment, complex drug regimen and side effects involved in the treatment of infectious diseases.

\section{REFERENCES}

Coudert P, Duroux E, Bastide P, Couquelet J, Tronche P. Synthesis and evaluation of the aldose reductase inhibitory activity of new diaryl pyridazine-3-ones. Journal of Pharmacy Belgium, 1991; 46(6):37580.

Barraja P, Diana P, Lauria A, Passannanti A, Almerico A, Minnei M: Docking of indolo- pyrrolo pyrimidines to DNA -interactive polycyclics from amino-indole/pyrroles and BMMA. Bioorganic Medicinal Chemistry, 1999; 7:1591-1596.

Chandra NS, Sanjib.B, Dipak.C, Synthesis and Antimalarial Screening of Some New Isoquine Analogues. International Journal of ChemTech Research, 209; 1(2), 322-328
Eman DA, Mustafa MEA, Suzan M, Malek AZ, Randa GN, Ehab QAM and Mohammad SM. Synthesis and Biological Activity of Some 3-(4-(Substituted)-piperazin-1-yl)cinnolines. Molecules, 2012; 17:227-239.

Gautam N, Chaurasia O.P: Synthesis antimicrobial and insecticidal activity of some new cinnoline based chalcones and cinnoline based pyrazoline derivatives, Indian Journal of chemistry (B), 2010; 49:830-835.

Garofalo AW, Adler M, Aubele DL, Bowers S, Franzini M, Goldbach E, Lorentzen C, Neitz RJ, Probst GD, Quinn KP, Santiago P, Sham HL, Tam D, Truong AP, Ye XM, Ren Z. Novel cinnoline-based inhibitors of LRRK2 kinase activity. Bioorganic Medicinal Chemistry Letter, 2013;23(1):71-4.

Kim HS, Miyake H, Arai M, Wataya Y. A potent antimalarial activity of 5-fluoroorotate in combination with sulfa mono methoxine against Plasmodium falciparum in vitro and Plasmodium berghei in mice. Parasitology International, 1998;47: 59-67.

Li S, Zhao Y, Wang K, Gao Y, Han J, Cui B, Gong P. Discovery of novel 4-(2-fluorophenoxy)quinoline derivatives bearing 4oxo-1,4-dihydrocinnoline-3-carboxamide moiety as c-Met kinase inhibitors. Bioorganic Medicinal Chemistry, 2013;21(11):2843-55.

Mojahidul I, Anees. Synthesis, antitubercular, antifungal and antibacterial activities of 6-substituted phenyl-2-(3'substituted phenyl pyridazin-6'-yl)-2, 3, 4,5-tetrahydropyridazin-3-one. Acta Poloniae Pharmaceutica, 2008; 65(3):353-362.

Ruchelman AL, Singh SK, Ray A, Wu X, Yang JM, Zhou N, Liu A, Liu LF, LaVoie EJ. 11H-Isoquino [4,3-c]cinnolin-12-ones; novel anticancer agents with potent topoisomerase I-targeting activity and cytotoxicity. Bioorganic Medicinal Chemistry, 2004;12(4):795-806

Sadaf Q, Schwalbe R, Moore LS, Goodwin AC, 2007. Editors. Macro and Micro dilution Methods of Antimicrobial susceptibility testing protocols, CRC Press, Taylor and Francis Group. 75-79.

Satyanarayana M, Feng W, Cheng L, Liu AA, Tsai YC, Liu LF, LaVoie EJ. Syntheses and biological evaluation of topoisomerase Itargeting agents related to 11-[2-(N,N-dimethylamino)ethyl]-2,3- 
dimethoxy-8,9-methylenedioxy $\quad-11 \mathrm{H}$-isoquino[4,3-c]cinnolin-12-one (ARC-31). Bioorganic Medicinal Chemistry, 2008;16(16):7824-31.

Scott DA, Dakin LA, Del Valle DJ, Diebold RB, Drew L, Gero TW, Ogoe CA, Omer CA, Repik G, Thakur K, Ye Q, Zheng X. 3amido-4-anilinocinnolines as a novel class of CSF-1R inhibitor. Bioorganic Medicinal Chemistry Letter, 2011;21(5):1382-4.

Tonk RK, Bawa S, Chawla G, Deora GS, Kumar S, Rathore V, Mulakayala N, Rajaram A, Kalle AM, Afzal O. Synthesis and pharmacological evaluation of pyrazolo[4,3-c]cinnoline derivatives as potential anti-inflammatory and antibacterial agents. European Journal of Medicinal Chemistry, 2012; 57:176-84.

Trager W, Jensen JB. Human malaria parasites in continuous culture. Science, 1976; 193(4254):673-5.

Vikas S, Darbhamulla S. Synthesis, characterization and biological activities of substituted cinnoline culphonamides. African Health Sciences, 2009;9(4):275-8.
Wentland, M. P.; S. C. Aldous, M. D. Gruett, Robert, G. Powles, W. Danz, Kristina M. Klingbeil, A.Danielle Peverly, Ronald G. The antitumor activity of novel pyrazoloquinoline derivatives. Bioorganic \& Medicinal Chemistry Letters, 1995, 5(4), 405-410.

\section{How to cite this article:}

Unnissa SH, Nisha N, Reddy GK. Synthesis and in Vitro Anti Microbial Evaluation Including Anti-Malarial Activity of Pyrazole Based Novel Cinnoline Derivatives. J App Pharm Sci, 2015; 5 (11): 121-126. 